\title{
Development of the digital economy in the context of sustainable competitive advantage
}

\author{
Tatyana Krasota ${ }^{1}$, Ruslan Bazhenov ${ }^{1, *}$, Umut Abdyldaeva ${ }^{2}$, Svetlana Bedrina ${ }^{3}$, and Irina \\ Mironova ${ }^{4}$ \\ ${ }^{1}$ Sholom-Alechem Priamursky State University, Shirokaya Str.,70A,679015 Birobidzhan, Russia \\ ${ }^{2}$ Kyrgyz State University of Construction, Transport and Architecture named after N. Isanov, \\ Maldybaeva Str.,34B,720020 Bishkek, Kyrgyzstan \\ ${ }^{3}$ Far Eastern Federal University, Sukhanova Str., 8, 690090 Vladivostok, Russia \\ ${ }^{4}$ Khabarovsk State University of Economics and Law, Tikhookeanskaya Str.,134, 680042 \\ Khabarovsk, Russia
}

\begin{abstract}
The objective of the research is to provide a scientific analysis of the development of the digital economy in the context of sustainable competitive advantage. The methodological basis of the study was the theoretical provisions of fundamental and practical developments in the digital economy, lying at the intersection of various technologies. Under the influence of digital forms of economic activity and the scientific and educational sphere, knowledge and education are turning from a luxury article into a productive force of society. Meanwhile, a global reach of digital technologies in the context of sustainable competitive advantage leads to a change in culture, traditional approaches to education, and serves the interests of the accelerated deployment of the digital economy. Managing the development of human capital as a basic engine for the development of the economic system is an absolute must for the integration of information technologies into modern society. Individuals and effective institutions are becoming a fundamental factor in the productivity, effective development, and formation of the digital economy.
\end{abstract}

\section{Introduction}

Changing economic resources through technology platforms is a feature of the digital economy. The digital economy covers everything related to information technology, software, mobile communications, and data transmission in a particular country [1].

The basic element of digital economic activity is the growth of labor productivity in new and traditional industries through the use of digital technologies in the processing and transmission of information based on information resources and modern mobile communications. Digital data processing and transmitting technologies promote the development of innovative technologies in the country. Associated with innovation, the technological revolution can dramatically change the structure of economic life. At the same time, the role and importance of the industries of knowledge-based services, information sources, processing, and transmission of digital data, which are already equated

${ }^{*}$ Corresponding author: r-i-bazhenov@yandex.ru 
to the main production factors of production, will increase. The priority mechanism for the functioning of the digital economy consists of innovation-oriented enterprises, industries, and sectors of the economy.

We believe that global best practices and basic fundamental stages play a great strategic role in the development of the digital economy in any state. The presentation of the scientific problem of correcting the trajectory of digital economic activity and choosing an alternative sustainable competitive advantage is relevant.

M.A. Galindo-Martin et al. analyze the theoretical and quantitative effects of digital transformation and digital dividends on entrepreneurship. They showed the broad advantages of using technology in the form of digital transformation [2]. It should be noted that M.F. Schweitzer et al. studied the digital revolution in the process of creating and developing a new good (product or service), as well as the role of information systems for improving labor productivity [3]. At the same time, in our opinion, quite significant issues of the stages of development of the digital economy, processing and transmission of information data remained not disclosed. S. Nambisan et al. devoted their article to the comprehension of important issues of modern challenges in total digitalization in the economy [4], however, as we think, the indicated issues are not addressed completely enough and require serious scientific disclosure and solution. P.O. De Pablos and J.E.L. Gayo considered the features of the use of information technology and the capabilities of the digital economy as a tool for ensuring sustainable logistics economic processes in a modern state [5]. Other researchers also made a scientific contribution to the development of the problem of the digital Russian economy [6, 7].

The subject of our research is the modern digital economy, as well as the study of the conditions for sustainable competitive advantage in modern Russia. The designated topic seems to be highly relevant and requires its further scientific solution at the theoretical and practical levels.

The objective of the research is to provide a scientific analysis of the development and formation of the digital economy in the context of a sustainable competitive advantage.

\section{Materials and Methods}

The methodological basis of the study was the theoretical provisions of fundamental and practical developments in the digital economy, lying at the intersection of various technologies [8,9]. Methods of scientific abstraction and structuring were used as tools.

\section{Results and Discussion}

Applied economic science already has a modern model for the transition to the digital economy. It can be divided into four basic fundamental platforms that form the basis of the digital economy: institutional environment; scientific and educational sphere; high-speed information infrastructure; and development and distribution of new technologies.

The institutional environment (formal and informal constraints) is a factor in the development of the digital economic activity. The rules of the game perform several important and necessary functions: accelerating digital development and education, promoting competition in the market, and strengthening and supporting stability and comfort in the social sphere of our state.

At the same time, a new productive system of resource and factor support of production is being formed. The digitalized scientific and educational sphere (SES) is crucial in the universal development and formation of human capital [7]. It allows for high-quality training for digital economy workers, especially in the field of innovation. There is a need 
to create conditions for sustainable competitive advantages of such training. Another equally important condition for implementation is the development of the state SES capable of forming an effective conglomeration of science, education, and business based on the goals of Russia's digital development. SES can function quite effectively in the structure of the digital economy, as it generates and forms new ideas, innovations, grants, etc., creates various competitive directions for the development of human capital. At the same time, digital technologies are modifying the content of the SES, leading to its ever-greater centralization. As a result, one can see an increase in the volume of scientific knowledge with subsequent implementation in production. Meanwhile, the possibilities of implementing the creative functions of digital economic activity are in the scientific and educational sphere, which is open to change, flexible, sensitive to original projects and actions, and able to promote the diffusion of information technologies.

Thus, the scientific and educational sphere is a conglomerate of scientific centers, research institutes, universities, which include high-tech structures that specialize in the creation and implementation of advanced technologies.

The sufficiently effective functioning of the scientific and educational sphere requires an appropriate level of information infrastructure of the state, which significantly affects the integration of universities, scientific organizations, businesses, and a general focus on common goals. This is a significant driver for increasing the activity of universities, academic institutions, and research centers.

K.J. Arrow notes that, as a result, "knowledge is not just a useful and necessary good, but an object of sale and purchase" [10]. S.G. Mikhneva believes that "knowledge, but not labor, is the primary source of improving labor efficiency" [11]. Based on digitalization, subjects of the scientific and educational sphere actively implement multiple possibilities of intellectual and educational clusters.

Human capital, its development, improved creativity and intelligence are becoming a factor in digital economic activity. Digital transformation contributes to the dominance of intellectual work. Of course, physical labor not only remains but its activity, efficiency, flexibility, adaptability increase. As a result of the influence of applied research, the transformative function of intellectual activity replaces the cognitive one.

We should note that digital technologies and platforms as a form of an individual's existence significantly change the ways of thinking and acquiring knowledge and skills. These technologies have a profound, pervasive, and revolutionary impact on people.

Analysis of open-source data showed that the main increase in employment in the Russian economy at the moment is accounted for by intellectual professions. The distribution of the quality of education among the employed is changing, and the share of professions, which require graduate and postgraduate education, is growing. Postgraduate education becomes a determining factor, carries a strategic factor.

The state employment policy is now based on active training and retraining of workers. The ability to perform various scientific and industrial, innovative tasks is becoming a fundamental factor.

There is a steady trend in digital economic activity in the development of the creative thinking of workers. Innovation and creativity play one of the main roles in ensuring sustainable competitive advantages $[12,13]$.

One of the priorities of the scientific and educational sphere is the formation of modern creative thinking of individuals, which acts as intellectual property. This skill gives the employee more opportunities in the digital economy. New achievements in science require a creative workforce, as the quality and content of intellectual work determine the future development of society $[14,15]$.

This trend has emerged from a change in the content of economic activity under the influence of digital technologies. There is a transition in modern Russian society from 
predominantly physical labor to predominantly intellectual labor. The structure of employment and the range of professions are changing, jobs implying intellectual functions are appearing [16].

Such an economic transformation caused by the growth and development of the digital economy forces the content, methods, technical means and forms of employee training to change. In the context of sustainable competitive advantage, the needs of the digital economy enhance the role and importance of the scientific and educational sphere $[17,18]$.

\section{Conclusions}

Thus, under the influence of digital forms of economic activity and the scientific and educational sphere, knowledge and education are turning from a luxury article into a productive force of society. Meanwhile, a global reach of digital technologies in the context of sustainable competitive advantage leads to a change in culture, traditional approaches to education, and serves the interests of the accelerated deployment of the digital economy.

Managing the development of human capital as a basic engine for the development of the economic system is an absolute must for the integration of information technologies into modern society.

Individuals and effective institutions are becoming a fundamental factor in the productivity, effective development, and formation of the digital economy.

\section{References}

1. P. Michelman, S. Narayen, Mit Sloan Management Review, 60 (2019)

2. M. A. Galindo-Martin, M. S. Castano-Martinez, M. T. Mendez-Picazo, Journal of Business Research, 101 (2019)

3. F.V. Schweitzer, M. Handrich, S. Heidenreich, International Journal of Innovation Management, 23 (2019)

4. S. Nambisan, M. Wright, M. Feldman, Research Policy, 48 (2019)

5. P. O. De Pablos, J. E. L. Gayo, Behaviour \& Information Technology, 38 (2019)

6. A. Davydov, V. Baranov, E. Sudov, Standards and quality (2002)

7. E. A. Gasanov, A. E. Zubarev, T. G. Krasota, Advances in Economics, Business and Management Research, 47 (2020)

8. M. Liu, S. Zha, W. He, TechTrends, 63 (2019)

9. A. N. Oleynik, Institutional aspects of socio-economic transformations (2000)

10. K. J. Arrow, Information and economic behavior (1973)

11. S. G. Mikhneva, Bulletin of RUDN University. Series: Economics (2002)

12. R. Foster, Innovation: The Attacker's Advantage (1986)

13. S.M. Menshchikov, L.A. Klimenko, Long waves in the economy. When society changes its skin (2016)

14. A. Young, P. A. Rogers, Mining Metallurgy \& Exploration, 36 (2019)

15. R. McKinnon, International Social Security Review, 72 (2019)

16. M. Herrmann, H. Truebel, J. P. Ehlers, P. Boehme, Geburtshilfe und Frauenheilkunde, 79 (2019)

17. A. L. Junge, Brazilian Journal of Operations \& Production Management, 16 (2019)

18. M. Zaki, Journal of Services Marketing, 33 (2019) 